\title{
Design and Development of Multi-rotorcraft-based Unmanned Prototypes of Personal Aerial Vehicle
}

\author{
Muljowidodo* \\ Center for Unmanned System Studies, Institut Teknologi Bandung, Indonesia \\ Agus Budiyono** \\ Smart Robot Center, Department of Aerospace-IT Engineering, \\ Konkuk University, Seoul, Korea
}

\begin{abstract}
The paper presents the design, development and testing activities of the multirotorcraft-based unmanned aerial vehicle at the Center for Unmanned System Studies, Institut Teknologi Bandung (ITB), Indonesia. The multi-rotor system was selected as the design stepping stone for future development of personal aerial vehicle prototypes. A step-by-step design program is conducted to study the technology building blocks and critical issues associated with the design, development and operation of personal aerial vehicles. A number of multi-rotor configurations have been investigated providing basic guidelines for developing a stable unmanned aerial platform. The benefit of the presently selected configuration is highlighted and some preliminary testing results are presented.
\end{abstract}

Key words : rotorcraft-based unmanned aerial vehicle, personal aerial vehicle

\section{Introduction}

The ideas of a personal aerial vehicle (PAV), by which ordinary people can travel to distant places easily and safely, has been around for at least 60 years [1]. With the increasing problem of ground traffic in many cities and the need to make air travel more effective and efficient, the ideas of PAV have been revisited by many research institutes. The fact that in the intervening time the PAV has yet to be adopted for broader market indicates that there exist critical barriers (economically and technologically) that need to be addressed. A report published at NASA in 1994 presented an overview of status and issues of personal aircraft. The suggested personal aviation vehicle discussed in this report was an automatic VTOL-capable affordable converticar which meets, for both the air and ground sides, all applicable safety, environmental and nuisance regulations in terms of collision avoidance/ survivability, noise, emissions, ground-vicinity operations and reliability as well as providing reasonable ride quality, all weather operation (in the same sense as current automobile operations) and minimal maintenance requirements/cost [2]. It was envisaged that requisite technologies exist which includes composite materials, advanced Wankel IC engines, the electronics revolution in terms of size/cost/capability of sensors/control systems/computing, the GPS system, the emerging global satellite-based personal communications systems, computational fluid mechanics and flow control.

\footnotetext{
* Director

** Assistant Professor

E-mail : agus@konkuk.ac.kr
}

Tel : +82-2-450-3817

Fax : +82-2-444-6670 


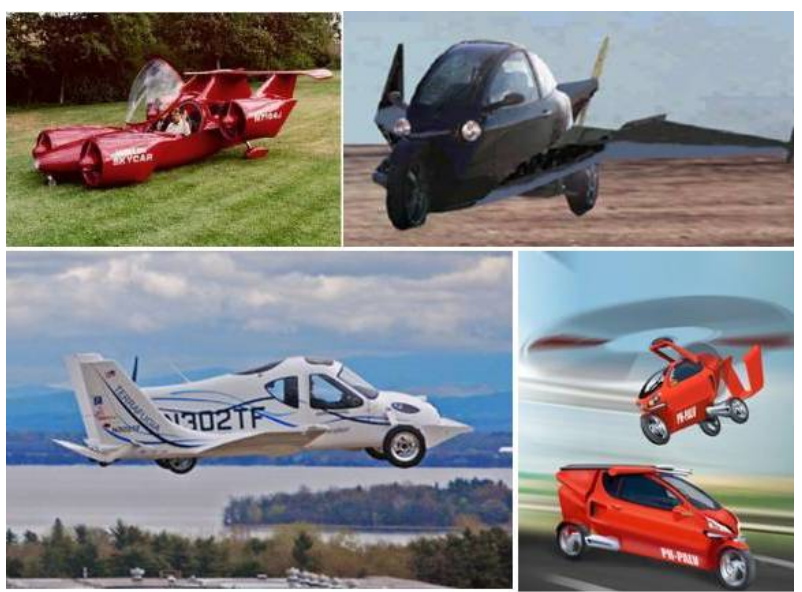

Fig. 1. Existing flying car concepts and products (source: [4], www.terrafugia.com and www.pal-v.com)

In [3] the author identified PAV as the viable future solution for a rural/regional and intra-urban transportation system. The bottom line for PAV technologies was that there is the opportunity to make small aircraft much better than they are today, and to develop an on-demand transportation system that would be much faster and provide more throughput than what exist today. This capability to travel faster, further, anytime, anywhere is a dream that is achievable, and one that could lead people into a new age of mobility. Some of the existing concepts and products of PAV is shown in Fig. 1.

Most of the current studies for PAV viabilities were conducted from the aerospace perspective. The authors in [4] presented the PAV technologies from the perspective of automotive industry. PAV was viewed as one of the fundamental new business opportunities in the automotive market: the tailored car (driven by production technology enablers, cars will be custom-made, not only by mixing and matching standard components, but by actually customizing the shape and style of components), brand worlds (car manufacturers extend their brands to other consumer product categories, attempting to 'immerse' consumers in a ' 'total brand experience' ' that includes, among others, financial, lifestyle, entertainment, and communication product) and multiple transportation modes (cars will extend other modes of individual transportation, such as water (boat-car), air (flying car), etc.).

The paper presents ongoing efforts in the design, development and testing of an unmanned prototype of personal aerial vehicle at Institut Teknologi Bandung, Indonesia. The paper is organized as follows. First, the underlying technologies and critical issues for the design of PAV are briefly surveyed in Section 2. The research activities at ITB for the design and development of PAV are presented in Section 3. The current proposed configuration of PAV is evaluated. Relevant research histories are summarized. Section 4 highlights the testing results of the current unmanned prototypes. Some issues in regards to the controllability of the vehicle are discussed. Concluding remarks end the paper.

\section{Technologies of Personal Aerial Vehicles}

$\checkmark \neg$ The design philosophy of PAV is different from that of general aviation vehicle. The PAV is typically designed by constraints rather than for performance. The design constraints include safety, ease of use (simple, low complexity system for ordinary people), operation (very low takeoff and landing speeds), environment (low emission, minimal noise, low downwash), cost and size. The technologies for PAV are aimed at achieving design within the above constraints. The following section summarizes the technology building blocks for the design of PAV. More complete discussions can be found in [2,3 and 5]. 


\subsection{Aerodynamics}

The aerodynamics problem of PAV stemmed from the need to provide an aerodynamic propulsive system that is simple enough, reliable and highly effective. The system should enable the PAV to take off and landing at a very low speed otherwise an efficient door-todoor concept of PAV cannot be realized. At NASA, for instance, the proposed approach is the use of circulation control highlift system (Coanda Jet Blowing).

\subsection{Aeroacoustics}

Emitted noise is the single greatest barrier to community acceptance of small aircraft today. A recent FAA comparison of aircraft noise showed that an average small aircraft today makes an equivalent perceived level of noise as commercial airliners [6]. Ducted propellers offer a method to significantly reduce the acoustical propagation characteristics through a combination of shielding, absorbing liners, elimination of asymmetric inflow, and the generation of higher frequency noise that dissipates more quickly in the atmosphere. The benefit of ducted propellers however needs to be traded-off with the reduced efficiency. Overall, the research in aeroacoustic is vital in bringing PAV into acceptable mass-products.

\subsection{Structure and materials}

The structure technology is aimed at developing a PAV that meets the size constraints while satisfying structural design criteria. To meet the size constraint a number of strategies have been proposed to limit the wing span which includes telescoping wing, folding wings and inflatable air-beam wings. Ultra lightweight structures are proposed for PAVs using aluminum, composite and hybrid materials. It must be noted that there is an inherent tradeoff between the need to reduce the weight for air operation and minimum body weight required for stability on the ground.

\subsection{Stability and Control}

The need for stability of PAV is derived from the same need for other type of aerial vehicles. The primary difference between PAV and general aviation vehicle is on the controllability and handling qualities issues. The PAV needs to have a higher degree of handling qualities since they are intended not for skilled pilots but ordinary people.

\subsection{Avionics}

Traditionally, handled by Air Traffic Control (ATC) where aircraft pilots are in radio communication with a human controller who routes aircraft using their positions and headings via radar tracks, transponder signals, etc. If widely operational PAVs are added into the density, the ATC would not be able to handle the traffic management. In a study in [7], it is envisaged that PAV navigation are comparable to the way on-demand automobile traffic navigation is accomplished. Each PAV is under its own control, and it follows certain "rules of the road" which allow other PAVs to cooperatively utilize the same airspace, similar to the way automobile traffic uses road networks. The avionics design for PAV should therefore allow the operation of the vehicle where there is no control towers, radar or approach lights. The PAV needs to incorporate supportive navigation such as built-in terrain mapping and airspace collision avoidance. 


\subsection{Power system}

Various types of engine have been studied and proposed for use to power the PAVs. The studies include the use of reciprocating engine, turbine engine, micro and mini turbine and electrical propulsion each with its own characteristic and limitation. A study at NASA reported in [8] summarizes the results of a first-order feasibility study for an all-electric personal air vehicle utilizing a fuel cell-powered propulsion system. A representative aircraft with an internal combustion engine was chosen as a baseline to provide key parameters to the study, including engine power and subsystem mass, fuel storage volume and mass, and aircraft range [8].

\subsection{Modeling and Analysis}

Continuous efforts in the design and development of PAV technologies have lead to the development of dedicated modeling and analysis tools. Recent development as surveyed in [6] is:

- Vehicle SketchPad concept to analysis modeler

- Haptic Naturalistic Flight Deck bi-directional experimental control stick and simulator

- Mobility analysis tool with lifecycle cost analysis and web-based analysis

- PAV manufacturing cost tool based on labor assembly hours

- Ducted propeller performance and noise tool

- Empirical circulation control aerodynamic prediction tool

- Multi-Gas Generator Fan cycle analysis tool

- Circulation control nacelle experiment methods

- Simajin agent-based high density simulation tool

- Skin-stiffened structure analysis procedure

\section{Design and Development of PAV Prototypes at ITB}

\subsection{Research program}

The research on PAVs at ITB is spanned from previous research program on the rotorcraft-based unmanned aerial vehicles (RUAVs). Various configuration of RUAVs have been investigated from the aerodynamic [9], structure, design [10-12], instrumentation [13], modeling [14-17] and control [18-20] point of view. The research in conjunction with PAV is broken down into four phases: propulsion and control devices research, PAV model design and manufacturing, PAV stability and performance analysis, and control system development. Fig. 2 depicts prototypes developed during for the research on the propulsion devices.

Fig. 3 shows the first PAV prototype and its outdoor testing.

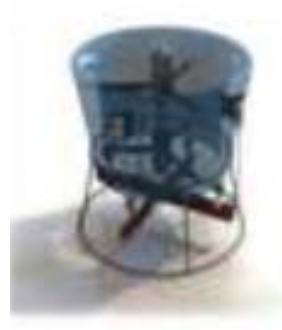

(a)

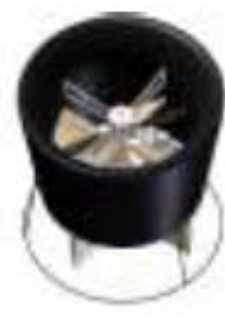

(b)

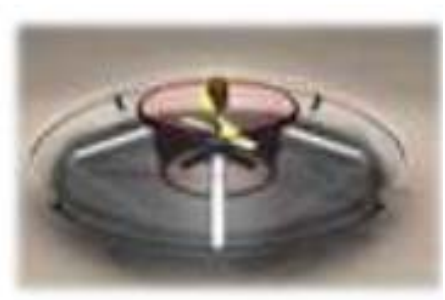

(c)

Fig. 2. Propulsion device prototypes: (a) Coaxial Rotor Ducted Fan,

(b) Single Rotor Ducted Fan, (c) Coaxial Rotor Ducted Fan with quadruple control channel 


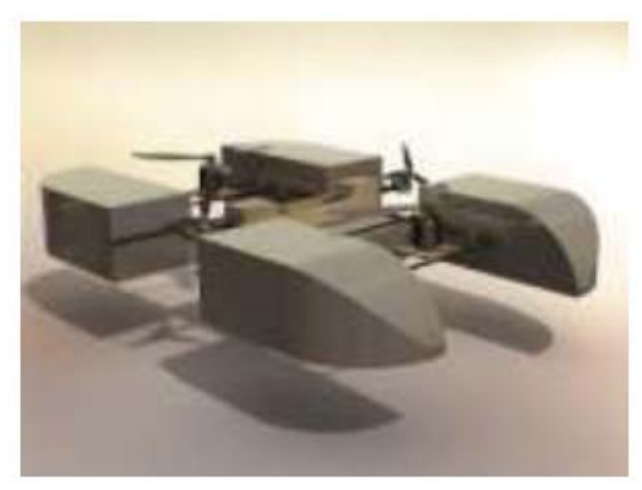

(a)

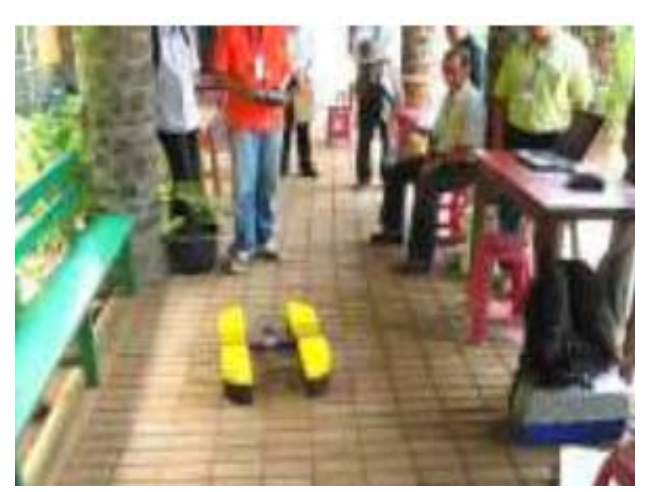

(b)

Fig. 3. First PAV prototype: (a) 3 D drawing (b) Testing

\subsection{ITB PAV design concepts}

There exist various design concepts for PAVs namely CTOL (dual-mode), STOL (using tail fan), SSTOL (using single spiral wing) and VTOL (tilt nacelle). Typically the reference conventional baselines for PAV are helicopter/autogyro/commercial air vehicle (for the aerial segment) and auto/taxi/rental car (for the ground segment). The advanced concepts for PAV can be categorized into single- mode and dual-mode configuration. Various prototypes have been developed at NASA Langley, Georgia Tech, CalPoly, Boeing and VPI. At ITB, two configurations are investigated. Fig. 4 depicts the proposed conventional take-off and landing dual mode PAV concept. The operation of this PAV will require a certain length of runway.

The second proposed configuration is shown in Fig. 5. The second configuration uses a multiple-rotor system. A main rotor provides the primary lift for takeoff and landing. Whereas the two side-rotors provide an attitude control. This VTOL PAV configuration will not require runway for take-off and landing therefore it provides more effective point-to-point travel.
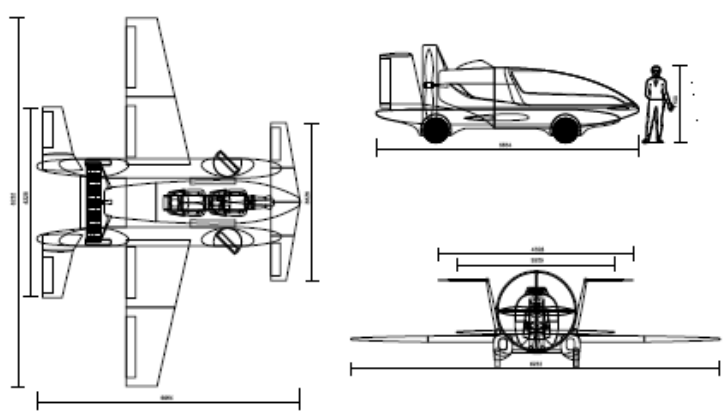

Fig. 4. ITB Conventional Takeoff-landing PAV

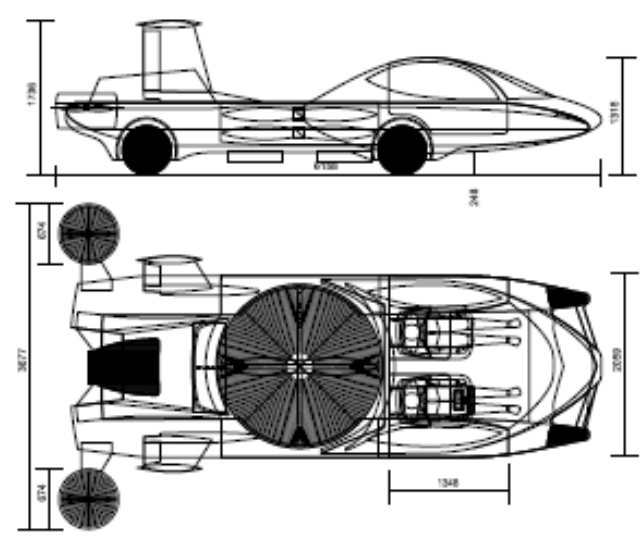

Fig. 5. ITB Vertical Takeoff-landing PAV

\subsection{ITB PAV unmanned prototypes}

An unmanned prototype was developed based on the VTOL configuration. The perspective drawing is shown in Fig. 6. The 3D images of the prototype are given in Fig. 7. Fig. 8 provides photos of the developed prototype. 

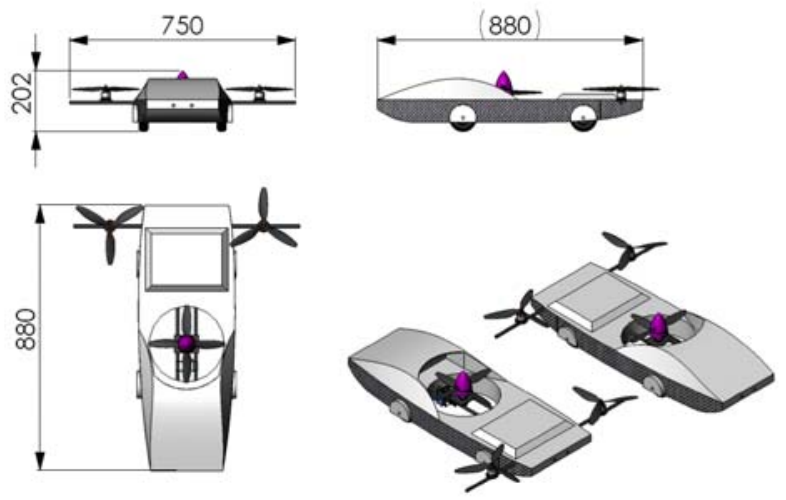

Fig. 6. ITB unmanned PAV prototype drawing
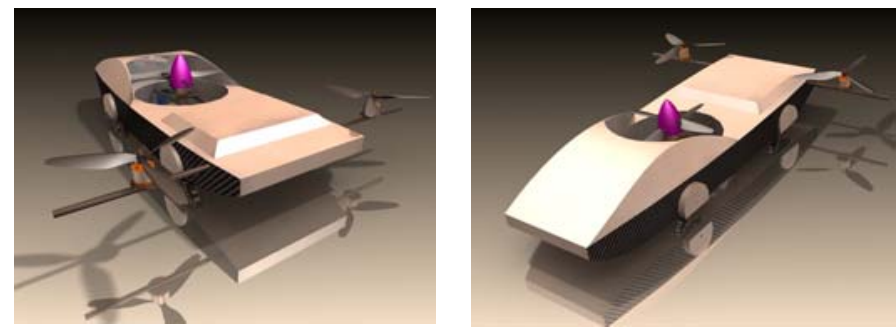

Fig. 7. ITB unmanned PAV prototype 3D images
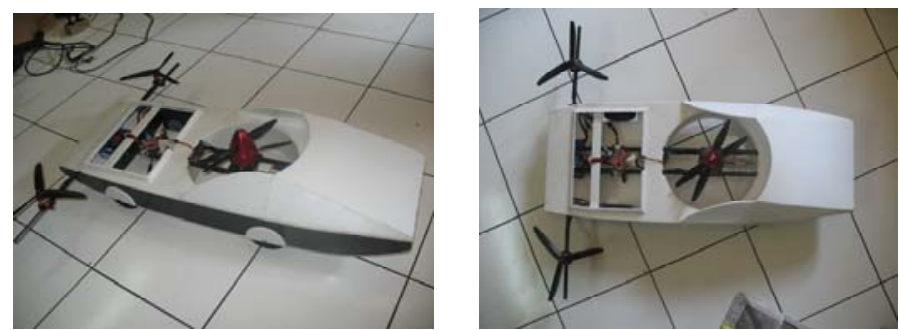

Fig. 8. ITB unmanned PAV prototype

\section{Testing of PAV Prototype}

\subsection{Attitude control}

The most important aspect in designing a multi-rotor vehicle is achieving stability. The stability can be achieved when there are ways to make forces and moments work in equilibrium. For the ITB multi-rotor unmanned PAV, the primary lift given by the main rotor is balanced by the two side-rotors in the longitudinal sense. The lateral forces and moments balances are provided by the balance of the two side-rotors.

The controllability of the unmanned PAV is attained by the differential rotor thrust. In the pitch channel, the pitch attitude can be achieved by the differential thruster between main rotor and two side-rotors. The roll control is obtained by the differential between left side rotor and right side rotor. The only non-differential control is the guide fin provided for yaw control. The installation of the guide fin and its alignment is shown in Fig. 9. The actual implementation is depicted in Fig. 10. 

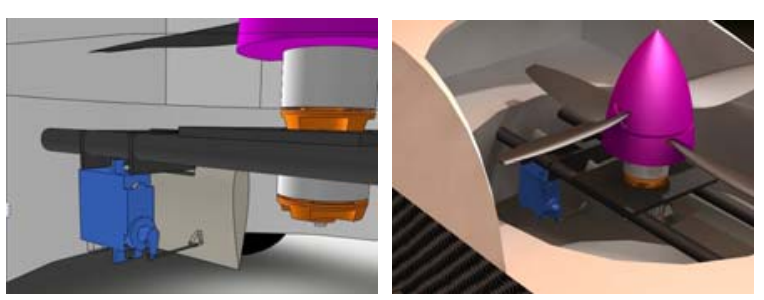

Fig. 9. Guide fin of ITB unmanned PAV prototype

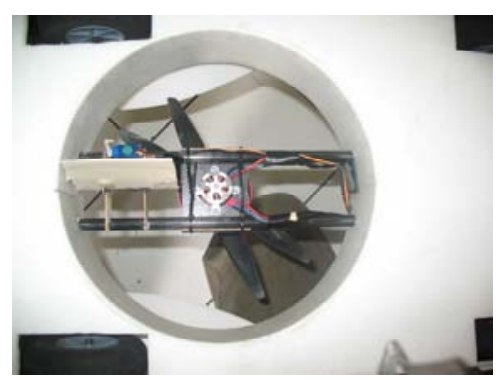

Fig. 10. Yaw control of ITB unmanned PAV prototype

\subsection{Indoor testing}

Due to the size, the unmanned PAV prototype is sensitive to wind disturbance. Therefore the flight testing was conducted in the indoor environment. The first phase of flight testing was to evaluate the controllability of the vehicle. The effectiveness of all control channels was tested. As in actual operation, the most critical segment of the PAV is take-off and landing phase. The hovering performance of the multi-rotor prototype is therefore considered as the most important aspect to be examined. During the testing period, the pilot could demonstrate a successful takeoff, followed by a controlled hover at about 1 meter from the ground for one minute and loitering flight for two minutes prior to a smooth landing. The controlled hover performance is shown in Fig. 11.

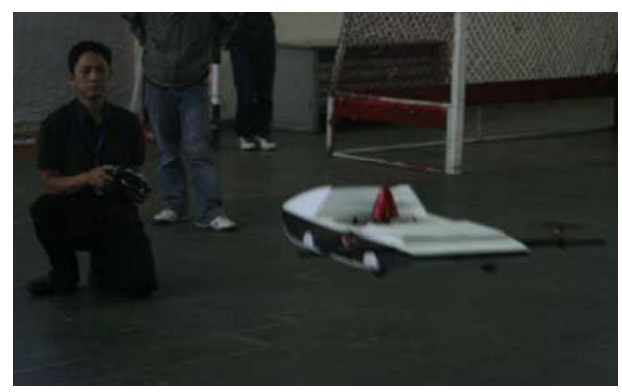

Fig. 11. Hover performance of ITB unmanned PAV

\section{Concluding Remarks}

The paper presents the design, development and testing activities of the multirotorcraft-based unmanned aerial vehicle at the Center for Unmanned System Studies, Institut Teknologi Bandung (ITB), Indonesia. Safety is considered as the most critical aspect for the operation of the PAV therefore the first phase of research is centered on the development and testing of the unmanned prototypes. The first multi-rotorcraft-based PAV has been successfully developed and tested. The controllability of the PAV was demonstrated through a successful flight cycle that includes take-off, hovering, loitering and landing. Future work will include the development of a larger prototype for the actual outdoor testing. The synthesis of an automatic flight control system is anticipated for the second prototype to improve the handling quality of the vehicle and to ease the load of the pilot.

\section{Acknowledgments}

The authors would like to acknowledge the support of the flight test pilot, Dian, and all supporting technical personnel in testing the unmanned PAV prototypes. The second author was supported by the MKE (Ministry of Knowledge Economy), Korea, under the 
ITRC Information Technology Research Center) support program supervised by the IITA (Institute for Information Technology Advancement) (IITA-2009-C1090-0902-0026.

\section{References}

1. A. S. Hahn, "Next Generation NASA GA Advanced Concept", NASA Langley Research Center Report. 2006.

2. Scott G. Anders, Scott C. Asbury, Kenneth S. Brentner, Dennis M. Bushnell, Christopher E. Glass, William T. Hodges, Shelby J. Morris, Jr., and Michael A. Scott, "The Personal Aircraft--Status and Issues", NASA Technical Memorandum 109174. December 1994.

3. M. D. Moore, "Personal Air Vehicles: A Rural/ Regional and Intra-Urban OnDemand Transportation System”, AIAA Paper 2003, 2003.

4. M. Seidel, C.H. Loch and S. Chalil, "Quo Vadis, Automotive Industry? A Vision of Possible Industry Transformations" , European Management Journal Vol. 23, No. 4, pp. 439 449, 2005.

5. D.N. Marvis and D. DeLaurentis, "Personal Air Vehicle Exploration Tool and Modeling”, NAS3-00179/70884-D, 2002.

6. M. D. Moore, “NASA Personal Air Transportation Technologies”, NASA Langley Research Center Report

7. RhinoCorps Ltd. Co," NASA PAV Simulation Effort”, Presented to NASA LARC, 2005.

8. L. L. Kohout and P.C. Schmitz, "Fuel Cell Propulsion Systems for an All-Electric Personal Air Vehicle”, NASA/TM 2003-212354, AIAA $2003 \quad$ 2867,2003

9. M. Kartidjo, S. A. Nugroho, and R.Parama, "Design and Testing Guide Vanes of Single Rotor Ducted Fan”, International Symposium on Intelligent Unmanned Systems (ISIUS2009), Jeju Island, June 2009.

10. A. Budiyono, "Design and Development of Autonomous Uninhabited Air Vehicles at ITB: Challenges and Progress Status”, Paper presented at the Aerospace Indonesia Meeting, Bandung, Indonesia 27 July 2005.

11. Muljowidodo, A. Budiyono, and Kurniantoro, "Development of VTOL-UAV", International Conference on Production and Manufacturing, Kuala Lumpur, Malaysia, March 2006.

12. Muljowidodo and A. Budiyono, "Design and Development of Micro Aerial Vehicle at ITB," International Conference on Technology Fusion, Seoul, Korea, 17-19 May 2006.

13. A. Budiyono, W. Adiprawita, and Muljowidodo, "MEMS-based Inertial Measurement Unit for Unmanned Aerial Vehicle Applications," 5th International Symposium of Nano Manufacturing, ISNM5, Singapore, January 23-25, 2008.

14. T. Sudiyanto, A. Budiyono, and H.Y. Sutarto, "Hardware In-the-Loop Simulation for Control System Designs of Model Helicopter," Paper presented at the Aerospace Indonesia Meeting, Bandung, Indonesia 27 July 2005.

15. Idris E. Putro, A.Budiyono, K.J.Yoon and D.H. Kim, "Modeling of Unmanned Small Scale Rotorcraft based on Neural Network Identification," IEEE International Conference on Robotics and Biomimetics, Bangkok, Thailand, February 22-24, 2009.

16. A. Budiyono, T. Sudiyanto, and H. Lesmana, "Global Linear Modeling of Small Scale Helicopter," In Intelligent Unmanned Systems: Theory and Applications. Studies in Computational Intelligence (SCI) series, Springer-Verlag, April, 2009.

17. Agus Budiyono, Kwang Joon Yoon, Finley D. Daniel, "Integrated identification modeling of rotorcraft-based unmanned aerial vehicle," med, pp. 898-903, 2009 17th Mediterranean Conference on Control and Automation, 2009.

18. A. Budiyono, "Onboard Multivariable Controller Design for a Small Scale Helicopter Using Coefficient Diagram Method," International Conference on Emerging System Technology, Seoul, Korea 19-20 May 2005.

19. A. Budiyono, and S.S. Wibowo, "Optimal Tracking Controller Design for A Small Scale Helicopter,” J Bionic Eng., 2007, 04 (04), 271-280.

20. A. Budiyono, and T. Sudiyanto, "Control of Small Scale Helicopter using s-CDM and LQ Design," In Intelligent Unmanned Systems: Theory and Applications. Studies in Computational Intelligence (SCI) series, Springer-Verlag, April, 2009. 